\title{
Aging, tumor suppression and cancer: high wire-act!
}

\author{
Judith Campisi ${ }^{\mathrm{a}, \mathrm{b}, *}$ \\ ${ }^{a}$ Life Sciences Division, Lawrence Berkeley National Laboratory, 1 Cyclotron Road, Berkeley, CA 94720, USA \\ ${ }^{\mathrm{b}}$ Buck Institute for Age Research, 8001 Redwood Blvd., Novato, CA 94545, USA
}

\section{Abstract}

Evolutionary theory holds that aging is a consequence of the declining force of natural selection with age. We discuss here the evidence that among the causes of aging in complex multicellular organisms, such as mammals, is the antagonistically pleiotropic effects of the cellular responses that protect the organism from cancer. Cancer is relatively rare in young mammals, owing in large measure to the activity of tumor suppressor mechanisms. These mechanisms either protect the genome from damage and/or mutations, or they elicit cellular responsesapoptosis or senescence-that eliminate or prevent the proliferation of somatic cells at risk for neoplastic transformation. We focus here on the senescence response, reviewing its causes, regulation and effects. In addition, we describe recent data that support the idea that both senescence and apoptosis may indeed be the double-edged swords predicted by the evolutionary hypothesis of antagonistic pleiotropyprotecting organisms from cancer early in life, but promoting aging phenotypes, including late life cancer, in older organisms.

(C) 2004 Published by Elsevier Ireland Ltd.

Keywords: Antagonistic pleiotropy; Apoptosis; Cellular senescence; Gene expression Pattern; p53; p16

\section{Introduction}

There have been extraordinary advances in the last decade in understanding the evolution of genomes and the genetic basis for aging. The idea that aging is under genetic control may now seem obvious, especially considering the sometimes large differences in life span among organisms with comparatively similar genomes (Williams, 1957). Recent discoveries, however, have explicitly identified evolutionarily conserved genes that are important regulators of life span, as well as early life fitness, among diverse species (Guarente and Kenyon, 2000; Kirkwood and Austad, 2000; Walker et al., 2000; Finch and Ruvkun, 2001). In general, these recent findings support modern evolutionary theories of aging. They have uncovered candidate genes on which evolution likely acted to produce species-specific life spans, and elucidated conserved pathways within organisms that link metabolism, reproduction and life span. At present, we still know remarkably little about the cellular and molecular bases for longevity differences among species.

* Tel.: +1 510486 4416; fax: +1 5104864545 .

E-mail address: jcampisi@lbl.gov.
However, owing in large measure to our recent understanding of the genetic similarities in longevity pathways among species, we are gaining important insights into the mechanisms that control aging within species.

Here, we review the evolutionary theory of antagonistic pleiotropy, and emerging evidence that aging in complex multicellular organisms is caused in part by the antagonistically pleiotropic effects of tumor suppressive mechanisms-mechanisms that evolved to prevent the development of cancer in young organisms.

\section{Evolution of life spans and cancer}

\subsection{Environment and the evolution of genomes}

An obvious tenet of any evolutionary theory is that hereditable traits, including species-specific life spans, are controlled by genes, and that these genes in turn evolved in response to environmental pressures. In addition, the environments in which genomes evolve are typically fraught with natural hazards-predators, infection, food scarcity, harsh climatic conditions, etc., which generally kill 
organisms long before they reach "old age". That is, the environments in which genomes evolve typically eliminate reproductively fit organisms at relatively young ages. Consequently, any gene mutation or multi-gene process that has deleterious albeit delayed effects-delayed with respect to the average age at which the environment eliminates organisms - will be retained. Thus, aging is thought to occur because phenotypes that have escaped the force of natural selection persist (Williams, 1957; Kirkwood and Austad, 2000; Finch and Ruvkun, 2001).

Consider, now, that genomes evolve relatively slowly, and that environments can change relatively rapidly. This is precisely what has happened to modern humans. We have, very rapidly on evolutionary time scales, eliminated many of the extrinsic hazards that dominated the environments in which our genome evolved. Parenthetically, we have also done this for our favorite animal models for biomedical research, at least within the confines of our animal colonies! Hence, aging - those delayed deleterious phenotypes that escaped the force of natural selection during evolution-is pervasive, especially among developed populations, which have been most successful in eliminating extrinsic hazards.

\subsection{Cancer and age-related disease}

Just as aging is considered by evolutionary biologists to be a consequence of the declining force of natural selection with age, age-related diseases can be considered phenotypes that have escaped the force of natural selection. Age-related diseases are generally considered to be degenerative in nature, a result of the overall decline in tissue structure and function that is a hallmark of aging. There are, however, exceptions. The most notable of these is cancer.

In simple terms, cancer can be considered a gain-offunction disease (discussed further below). That is, cells must acquire functions (e.g., hyperproliferation, resistance to cell death, migratory and invasive properties) in order to give rise to malignant tumors. Of particular interest, cancer is not a universal feature of aging. There are many organisms, including some of our favorite organisms for studying aging (Drosophila melanogaster; Caenorhabditis elegans), which never develop cancer. Here, we define cancer as an ectopic mass of supernumerary cells that develops postnatally and has the potential to kill the organism. What distinguishes organisms that do and do not develop cancer? One obvious distinction is the presence of renewable somatic tissues-somatic tissues that contain dividing or division-competent cells-in the adults. Thus, cell division in adult somatic tissues appears to put organisms at risk for developing cancer. This is not surprising, given that we now know that DNA replication puts cells at endanger for acquiring and fixing mutations (Kunkel and Bebenek, 2000; van Brabant et al., 2000; Friedberg et al., 2002; Thompson and Schild, 2002), and that somatic mutations are a major cause of cancer (Bishop,
1995; Simpson and Camargo, 1998; Gray and Collins, 2000; Knudson, 2000).

Why would evolution select for organisms with renewable tissues, given the danger of developing cancer? The benefits of renewable tissues - the ability to regenerate or repair tissues damaged by injury or endogenous degenerative processes - may have outweighed their risks, in conjunction with the co-evolution of mechanism to suppress cancer (discussed below). Moreover, we speculate that the evolution of renewable tissues afforded organisms long life spans.

\section{Tumor suppressor mechanisms}

Because cell division can lead to mutations and hence cancer, organisms with renewable tissues had to evolve strategies to prevent the cancer. Collectively, these strategies are termed tumor suppressor mechanisms. Tumor suppressor mechanisms can, in general, be broadly classified into two major categories - caretakers and gatekeepers (Kinzler and Vogelstein, 1997).

Caretaker tumor suppressors act on the genome, generally by preventing or repairing DNA damage. Thus, caretaker tumor suppressors restrain the development of cancer by suppressing the development of mutations. Since mutations not only cause cancer, but have also been proposed to independently contribute to aging, genes that encode caretaker tumor suppressor functions are straightforward longevity assurance genes (Martin, 1966; Dolle et al., 2002; Vijg and Dolle, 2002; Hasty et al., 2003).

Gatekeeper tumor suppressors, by contrast, act on cells, causing them to die (apoptosis) or permanently arrest proliferation (senescence). Thus, gatekeeper tumor suppressors restrain the development of cancer by eliminating or preventing the growth (used here interchangeably with growth) of potential cancer cells. In contrast to the caretakers, we suggest that genes encoding gatekeeper tumor suppressors have both beneficial (anti-cancer) and deleterious (pro-aging) effects, depending on the age of the organism. That is, gatekeeper tumor suppressors are an example of evolutionary antagonistic pleiotropy, which, as discussed below, is hypothesized to explain at least in part why organisms age.

\section{Antagonistic pleiotropy}

Because aging is a consequence of the declining force of natural selection with age, traits that benefit young organisms-suppressing cancer, for example-can have unselected deleterious effects-driving aging phenotypes, for example-later in the life span. This is the concept of evolutionary antagonistic pleiotropy (Williams, 1957; Rose, 1991; Kirkwood and Austad, 2000; Finch and Ruvkun, 2001). In simple terms, antagonistic pleiotropy holds that what is good for an organism when it is young can be bad for 
it when it is old. Antagonistic pleiotropy occurs because genes with the "good" attributes of optimizing the fitness of young organisms evolved under the pressure of natural environments, in which, as discussed above, extrinsic hazards are high and old organisms are relatively rare. If the same genes have "bad" attributes, but their manifestation is delayed - that is, manifest only after most of the population has been eliminated by extrinsic hazards-they cannot be eliminated by natural selection. Hence, genes with delayed deleterious actions (antagonistic pleiotropy) can persist. The consequences of their deleterious effects, then, are abundantly evident only in populations in which the hazardous environmental pressures have eased rapidly relative to the pace at which genomes evolve-the situation humans in the developed world (and mice in our animal colonies) now face.

How might the gatekeeper tumor suppressor genesthose that control apoptosis and cell senescence-be antagonistically pleiotropic?

Apoptosis prevents cancer by virtually eliminating cells that are damaged or otherwise potentially oncogenic (Reed, 1999; Green and Evan, 2002; Hickman et al., 2002). On the other hand, apoptosis can eventually deplete tissues of their constituent cells and/or deplete the stem cell pools that replenish renewable tissues (Joaquin and Gollapudi, 2001; Weinstein and Ciszek, 2002; Zhang and Herman, 2002; Campisi, 2003a, 2003b). Hence, with increasing age, apoptosis might cause an overall loss of tissue structure and function, a hallmark of aging. Apoptosis may be an especially important contributor to the degenerative diseases of aging.

Along the same lines of reasoning, cellular senescence prevents cancer by arresting the growth of potentially oncogenic cells (Sager, 1991; Bringold and Serrano, 2000; Lundberg et al., 2000; Reddel, 2000; Campisi, 2001). However, with increasing age, senescent cells, which are incapable of regeneration and show marked changes in function (discussed below), can accumulate. Again, this accumulation can lead to an overall loss of tissue structure and function (Campisi, 1996, 2003a, 2003b; Smith and Pereira-Smith, 1996; Faragher, 2000). Like apoptosis, cellular senescence may contribute to the degenerative diseases of aging. In addition, because the secretory phenotype of senescent cells can alter the local tissue milieu, senescent cells may also contribute to the hyperproliferative diseases of aging, including-ironically_cancer (Campisi, 1997, 2003a, 2003b).

At present, very little is known about what determines whether mammalian cells recover, die or senesce in the face of damage or stress. Some cell types-for example, T cells-are more prone to undergo apoptosis than senescence, whereas the opposite is true for other cell types-for example, fibroblasts. In addition, the level and type of stress may determine whether cells undergo an apoptotic or senescence response. Whatever the case, at least in mammals, both apoptosis and senescence important defenses against the development of cancer, yet both processes have the potential to be deleterious with time, and hence in older organisms.

Here, we review the evidence that the gatekeeper tumor suppressors that control apoptosis and senescence may be antagonistically pleiotropic. We focus our discussion primarily on the mammalian senescence response, which arguably has more complex age-related consequences in vivo than the apoptotic response, and for which there is mounting evidence for a role in aging. However, it is important to bear in mind that parallel arguments may hold for apoptosis.

\section{The senescence response}

\subsection{Causes of senescence}

The senescence response was first formally described as the process that limits the proliferation of human cells in culture (Hayflick, 1965). We now know that this limit is due in large measure to the loss of telomeric DNA that occurs when cells that do not express telomerase undergo DNA replication (Levy et al., 1992; Wright and Shay, 2001). Telomeres, the DNA sequence and proteins that cap the ends of linear chromosomes, are essential chromosomal elements, loss of which causes genomic instability, an enormous risk factor for malignant transformation (Artandi and DePinho, 2000; Shay and Wright, 2001; Kim et al., 2002; Blasco, 2003). Thus, the senescence response to short dysfunctional telomeres serves to arrest the growth of cells in danger of genomic instability, consistent with its role in tumor suppression.

In the last decade, it has become clear that many events and stimuli in addition to telomere dysfunction-all of which put cells at risk for neoplastic transformation-can induce a senescence response. These events include DNA damage (DiLeonardo et al., 1994; Chen et al., 1995; Robles and Adami, 1998), as well as perturbations to chromatin organization (Ogryzko et al., 1996; Jacobs et al., 1999; Itahana et al., 2003; Narita et al., 2003). They also include the expression of certain oncogenes (Serrano et al., 1997; Zhu et al., 1998; Dimri et al., 2000) that deliver supraphysiological mitogenic signals to cells, and the overexpression of certain tumor suppressor genes (Sugrue et al., 1997; McConnell et al., 1998; Dai and Enders, 2000; Dimri et al., 2000; Beausejour et al., 2003). The most potent tumor suppressor genes that induce senescence when overexpressed are those that encode components of the $\mathrm{p} 53$ and pRB tumor suppressor pathways, both of which are crucial for the senescence response (Shay et al., 1991; Bringold and Serrano, 2000; Lundberg et al., 2000; Campisi, 2001; Itahana et al., 2001).

\subsection{The senescent phenotype}

The senescence response is not a simple arrest of cell proliferation. Rather, senescent cells adopt a complex phenotype that entails many changes in gene expression 
(Cristofalo and Pignolo, 1993; Campisi et al., 1996; Faragher, 2000; Krtolica and Campisi, 2002). In addition to imposing a block to cell cycle progression, the senescence response causes changes in cell morphology (generally, adoption of an enlarged flattened shape). It also renders many (although not all) cell types resistant to apoptotic signals. Furthermore, senescent cells acquire celltype specific functional changes. Thus, senescent cells fail to proliferate, but also become resistant to elimination by apoptosis and do not function normally. We hypothesize that the resistance to apoptosis may explain why senescent cells accumulate, while their altered function may explain how they contribute to aging and age-related disease.

What are the functional changes that accompany the senescence response? These changes have been best characterized in fibroblasts, the cell type that synthesizes and maintains the stroma, the structure that underlies the cells of epithelial tissues and regulates their function (Donjacour and Cunha, 1991). Of particular interest, senescent human fibroblasts develop a secretory phenotype characterized by increased secretion of extracellular matrix remodeling enzymes, inflammatory cytokines and epithelial growth factors (Campisi, 1996; Faragher, 2000; Krtolica and Campisi, 2002). These secreted molecules can, at least in principle, have a field effect-altering the microenvironment of the surrounding tissue with respect to structure, inflammation status and epithelial function.

Because the senescent phenotype entails functional changes that can alter tissue structure and function, senescent cells - as they accumulate-may progressively promote the decline in tissue structure and function that characterizes aging. It is in this way that the senescence response may be antagonistically pleiotropic-protecting organisms from cancer at young ages, but promoting aging phenotypes at old ages. How much evidence is there for this idea?

\section{Testing the hypothesis that gatekeeper tumor suppressors, specifically the senescence response, is antagonistically pleiotropic}

The hypothesis that gatekeeper tumor suppressors, and particularly the senescence response, is antagonistically pleiotropic makes a number of predictions, not all of which have been tested experimentally. Here, we review the major predictions and, where applicable, the pertinent experimental results.

\subsection{Do senescent cells exist and accumulate with age in vivo?}

A prime prediction of the above hypothesis is that senescent cells exist and accumulate with age in mammalian tissues. This appears to be the case, with the important caveat that at present we have very few markers with which to identify senescent cells. In addition to the enlarged senescent morphology, one marker that is widely used is a neutral ( $\mathrm{pH}$ 6) $\beta$-galactosidase, termed the senescenceassociated $\beta$-galactosidase (SA-Bgal) (Dimri et al., 1995). The expression of this enzyme correlates strongly, although not exclusively, with the induction of senescence by any of the known senescence-inducing stimuli in a variety of cell types in culture. Because SA-Bgal is easily detected in situ by histochemical staining, it has been used to search for cells with senescent characteristics in vivo. Indeed, such (SABgal-positive) cells have been found in several tissues from humans and rodents. More important, their frequency has been shown to rise with increasing age in human skin, monkey skin and retina, human prostate, rodent kidney, human liver and human vascular endothelium (Dimri et al., 1995; Mishima et al., 1999; Pendergrass et al., 1999; Choi et al., 2000; Ding et al., 2001; Paradis et al., 2001; Vasile et al., 2001; Melk et al., 2003). Moreover, as discussed below, cells with senescent characteristics have been found at sites of age-related pathology, including atherosclerotic plaques and benign and premalignant lesions of the liver and prostate.

While these studies constitute little more than a 'smoking gun', they at least suggest that senescent cells appear to be present at the predicted times and locations.

\subsection{Do gatekeeper tumor suppressors promote aging?}

Another prediction is that gatekeeper tumor suppressors should promote aging. As noted earlier, genes encoding two tumor suppressor proteins - p.53 and $\mathrm{pRB}$ - are pivotal for establishing and maintaining cellular senescence. p53, a multifunctional transcriptional regulator (Sherr, 1998; Prives and Hall, 1999; Ryan et al., 2001; Wahl and Carr, 2001; Hofseth et al., 2004), is of particular interest because it is dispensable for mammalian embryogenesis and postnatal development, but crucial for preventing cancer. Indeed, most, if not all, malignant tumors harbor mutations in $\mathrm{p} 53$ or one of its critical regulators. p53 is also a quintessential gatekeeper tumor suppressor because it is a crucial regulator of both the apoptotic and senescence responses.

Recently, two groups created mouse models with constitutively hyperactive p53 (Tyner et al., 2002; Maier et al., 2004). p53 acts as a tetramer (Prives and Hall, 1999). In both mouse models, a truncated form of p53 was ubiquitously expressed, and the truncated forms were thought to form mixed tetramers with wild-type p53. Moreover, indirect evidence indicated that the mixed tetramers were hyperactive relative to tetramers composed solely of wild-type p53.

Consistent with p53's role as a potent tumor suppressor, the mutant mice were strikingly resistant to cancer. Since cancer is a major cause of death in laboratory mice, one might expect the mutant mice to be long-lived. This was not the case, however. Rather, both mutant mouse strains had a modestly shorter life span and displayed multiple signs of premature aging! Moreover, cells from these mice were more prone to undergo apoptosis (Tyner et al., 2002) or 
senescence (Maier et al., 2004) when stressed in culture Thus, hyperactive p53 conferred enhanced protection from cancer, but at the cost of accelerated aging, and the accelerated aging was associated with heightened sensitivity to apoptosis and senescence.

How might hyperactive 53 might cause accelerated aging? At least a partial answer to this question was provided by analysis of the mouse model created by Maier et al. In these animals, p53 appeared to upregulate components of the IGF-1 signaling pathway, which delivers mitogenic, survival and metabolic signals to mammalian cells. Supraphysiological IGF-1 signaling, in turn, stimulated a senescence response in cells from these mice. This finding is significant because components of the IGF-1/insulin pathway are among the evolutionarily conserved genes that have been shown to be important positive regulators of aging in diverse species (Guarente and Kenyon, 2000; Finch and Ruvkun, 2001; Bluher et al., 2003; Holzenberger et al., 2003; Rincon et al., 2004).

Together, these mouse models indicate that hyperactive tumor suppression by p53 promotes apoptosis and cell senescence and accelerates aging, supporting the idea that gatekeeper tumor suppressor functions are antagonistically pleiotropic (Campisi, 2004).

\subsection{Do senescent cells promote age-related pathology?}

A third prediction is that senescent cells should promote or accelerate the development of age-related pathology.

Although cancer is often studied independent of age, it is in fact a major age-related disease among mammals, age being the largest single risk factor for its development (Miller, 1991; DePinho, 2000; Balducci and Beghe, 2001; Campisi, 2003a, 2003b). The age-dependence with which cancer develops is sometimes attributed to the fact that cancer requires the accumulation of multiple mutations (Bishop, 1995; Simpson and Camargo, 1998; Gray and Collins, 2000; Knudson, 2000), which takes time. However, there is increasing evidence that mutation accumulation alone cannot fully explain why cancer incidence rises so sharply with age. What else is required for the development of cancer? Several decades of cell biology have established that many cells with oncogenic mutations also require a permissive tissue microenvironment in which to progress into a malignant tumor (DePinho, 2000; Park et al., 2000; Bissell and Radisky, 2001; Liotta and Kohn, 2001; Coussens and Werb, 2002). Of particular importance for the development of epithelial tumors - the major type of age-related cancer that develops in humans - are the interactions between the epithelium and the underlying stroma (Birchmeier and Birchmeier, 1995; DePinho, 2000; Bissell and Radisky, 2001; Chrenek et al., 2001; Liotta and Kohn, 2001; Tlsty and Hein, 2001).

Recent studies show that senescent stromal fibroblasts can stimulate the hyperproliferation of premalignant, but not normal, epithelial cells in culture (Krtolica et al., 2001; Dilley et al., 2003). Moreover, senescent fibroblast can stimulate the tumorigenic conversion of premalignant epithelial cells into frankly malignant tumors in vivo (Krtolica et al., 2001). The phenotype of senescent fibroblasts, described above, strongly resembles that of "activated stroma" or carcinoma-associated fibroblasts, both which have been shown to strongly stimulate tumor progression in cell culture models and in vivo (Skobe and Fusenig, 1998; Olumi et al., 1999; Shekhar et al., 2001; Martens et al., 2003). Interestingly, irradiated fibroblasts, which were likely senescent albeit not explicitly characterized as such, were also shown to promote epithelial tumor progression in vivo (Barcellos-Hoff and Ravani, 2000).

In many of these cases, the cancer-promoting activity of the senescent or activated stroma was due at least in part to their secretory phenotype (Skobe and Fusenig, 1998; Olumi et al., 1999; Krtolica et al., 2001; Shekhar et al., 2001; Martens et al., 2003; Parrinello et al., submitted for publication). Thus, the age-dependent accumulation of senescent cells, particularly senescent stromal cells, may synergize with the age-dependent accumulation of mutations, resulting in the rise in epithelial cancers. This idea is consistent with the identification of cells with senescent characteristics at sites of hyperplastic and premalignant lesions (Choi et al., 2000; Paradis et al., 2001; Castro et al., 2003). It is ironic indeed that an effective early life tumor suppressor mechanism (cellular senescence) can fuel the development of cancer late in life (Campisi, 2003a, 2003b)!

Much less is known about whether or to what extent the presence of senescent cells contribute to other age-related pathologies. Cells that express SA-Bgal and lack expression of thymosin- $\beta 10$, characteristics of senescent endothelial cells in culture have been found at site of atherosclerosis in human aorta (Vasile et al., 2001). Given that senescent cells secrete inflammatory cytokines, and that inflammation is thought to be an important contributory factor in atherogenesis, this result is consistent with the idea that senescent cells can initiate or promote age-related vascular disease. Similar types of evidence have implicated senescent cells in the etiology and/or progression of kidney fibrosis and age-related kidney dysfunction (Ding et al., 2001; Melk et al., 2003), osteoarthritis of the joints (Martin and Buckwalter, 2003) and venous ulcers of the lower extremities (Stanley and Osler, 2001).

Taken together, these findings support the idea that senescent cells can promote age-related pathologies, but the data thus far are of course still correlative and indirect. In some cases, cell culture and limited in vivo data indicate that senescent cells can promote pathological phenotypes. In other cases, senescent cells appear to be a 'smoking gun' by virtue of their presence.

6.4. Does reversal of the senescent phenotype or elimination of senescent cells prevent or ameliorate age-related pathology?

At present, we know very little about how to reverse the senescent phenotype or how to eliminate senescent cells in 


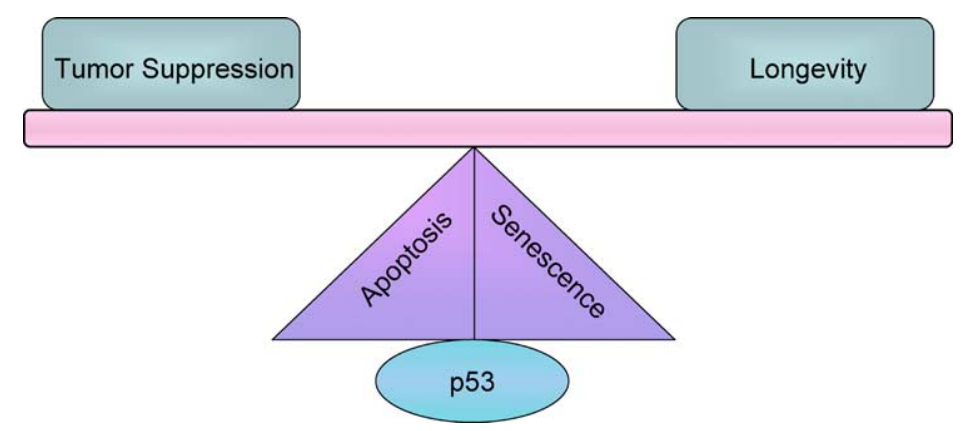

Fig. 1. Tumor suppression and longevity are balanced. In complex organisms such as mammals, we suggest that mechanisms that protect against cancer are balanced against those that ensure longevity. Specifically, the gatekeeper tumor suppressors such as p53, which regulate apoptosis and the senescence of replicative cells, are antagonistically pleiotropic, suppressing cancer in young organisms but promoting aging-including late life cancer-in old organisms.

vivo. We do know it is possible to reactivate the growth of some types of senescent cells by inactivating p53 (Gire and Wynford-Thomas, 1998; Beausejour et al., 2003). Cells that express the p16 tumor suppressor protein, which activates the $\mathrm{pRB}$ tumor suppressor, are refractory to reversal by p53 inactivation (Beausejour et al., 2003). We do not yet know how prevalent the p16 block to senescence reversal is in vivo. Whatever the case, inactivation of p53 is not a practical means for reversing the effects of senescent cells in vivo, as this will surely increase the incidence of cancer.

\section{Summary, conclusions and challenges}

We hypothesize that mechanisms that protect complex organisms from cancer (gatekeeper tumor suppressors) do so in balance against the mechanisms that promote longevity (Fig. 1). These cancer protection mechanisms engage the cellular processes of apoptosis and senescence. Both apoptosis and senescence are crucial for suppressing malignant tumorigenesis, yet both have the potential to contribute to aging and age-related pathology. The senescence response is notable in that the senescent phenotype may, ironically, also promote cancer at advanced ages. Critical testing of the hypothesis that the senescence response is antagonistically pleiotropic faces the challenge of developing ways to reverse the senescent phenotype without reversing the growth arrest, or switching the senescence response to an apoptotic response. If the hypothesis is correct, however, we face an even larger challenge-how to develop practical interventions that will minimize the pro-aging actions of gatekeeper tumor suppressor genes, while maintaining protection from cancer. The path to meeting this challenge is not yet clear, but will likely emerge as we develop a deeper understanding of how the senescent phenotype is established and maintained.

\section{Acknowledgements}

As always, thanks to past and present lab members for their refreshing ideas and hard work, colleagues for stimulating discussions and valuable reagents, and the National Institute on Aging, Department of Defense and University of California Breast Cancer Research Programs, Ellison Medical Foundation and Department of Energy for research support.

\section{References}

Artandi, S.E., DePinho, R.A., 2000. A critical role for telomeres in suppressing and facilitating carcinogenesis. Curr. Opin. Genet. Dev. $10,39-46$.

Balducci, L., Beghe, C., 2001. Cancer and age in the USA. Crit. Rev. Oncol. Hematol. 37, 137-145.

Barcellos-Hoff, M.H., Ravani, S.A., 2000. Irradiated mammary gland stroma promotes the expression of tumorigenic potential by unirradiated epithelial cells. Cancer Res. 60, 1254-1260.

Beausejour, C.M., Krtolica, A., Galimi, F., Narita, M., Lowe, S.W., Yaswen, P., Campisi, J., 2003. Reversal of human cellular senescence: roles of the p53 and p16 pathways. EMBO J. 22, 4212-4222.

Birchmeier, W., Birchmeier, C., 1995. Epithelial-mesenchymal transitions in development and tumor progression. EXS 74, 1-15.

Bishop, J.M., 1995. Cancer: The rise of the genetic paradigm. Genes Dev. 9, 1309-1315.

Bissell, M.J., Radisky, D., 2001. Putting tumours in context. Nature Rev. Cancer 1, 46-54.

Blasco, M.A., 2003. Mammalian telomeres and telomerase: why they matter for cancer and aging. Eur. J. Cell Biol. 82, 441-446.

Bluher, M., Kahn, B.B., Kahn, C.R., 2003. Extended longevity in mice lacking the insulin receptor in adipose tissue. Science 299, 572-574.

Bringold, F., Serrano, M., 2000. Tumor suppressors and oncogenes in cellular senescence. Exp. Gerontol. 35, 317-329.

Campisi, J., 1996. Replicative senescence: an old lives tale? Cell 84, $497-$ 500.

Campisi, J., 1997. Aging and cancer: the double-edged sword of replicative senescence. J. Am. Geriatr. Soc. 45, 1-6.

Campisi, J., 2001. Cellular senescence as a tumor-suppressor mechanism. Trends Cell Biol. 11, 27-31.

Campisi, J., 2003a. Cancer and ageing: rival demons? Nature Rev. Cancer 3, 339-349.

Campisi, J., 2003b. Cellular senescence and apoptosis: how cellular responses might influence aging phenotypes. Exp. Geront. $38,5-11$.

Campisi, J., 2004. Fragile fugue: p53 in aging, cancer and IGF signaling. Nature Med. 10, 231-232.

Campisi, J., Dimri, G.P., Hara, E., 1996. Control of replicative senescence. In: Schneider, E., Rowe, J. (Eds.), Handbook of the Biology of Aging. Academic Press, New York, pp. 121-149. 
Castro, P., Giri, D., Lamb, D., Ittmann, M., 2003. Cellular senescence in the pathogenesis of benign prostatic hyperplasia. Prostate 55, 30-38.

Chen, Q., Fischer, A., Reagan, J.D., Yan, L.J., Ames, B.N., 1995. Oxidative DNA damage and senescence of human diploid fibroblast cells. Proc. Natl. Acad. Sci. U.S.A. 92, 4337-4341.

Choi, J., Shendrik, I., Peacocke, M., Peehl, D., Buttyan, R., Ikeguchi, E.F., Katz, A.E., Benson, M.C., 2000. Expression of senescence-associated beta-galactosidase in enlarged prostates from men with benign prostatic hyperplasia. Urology 56, 160-166.

Chrenek, M.A., Wong, P., Weaver, V.M., 2001. Tumour-stromal interactions. Integrins and cell adhesions as modulators of mammary cell survival and transformation. Breast Cancer Res. 3, 224-229.

Coussens, L.M., Werb, Z., 2002. Inflammation and cancer. Nature 420, 860867.

Cristofalo, V.J., Pignolo, R.J., 1993. Replicative senescence of human fibroblast-like cells in culture. Physiol. Rev. 73, 617-638.

Dai, C.Y., Enders, G.H., 2000. p16 INK4a can initiate an autonomous senescence program. Oncogene 19, 1613-1622.

DePinho, R.A., 2000. The age of cancer. Nature 408, 248-254.

DiLeonardo, A., Linke, S.P., Clarkin, K., Wahl, G.M., 1994. DNA damage triggers a prolonged p53-dependent $\mathrm{G} 1$ arrest and long-term induction of Cip1 in normal human fibroblasts. Genes Dev. 8, 25402551.

Dilley, T.K., Bowden, G.T., Chen, Q.M., 2003. Novel mechanisms of sublethal oxidant toxicity: induction of premature senescence in human fibroblasts confers tumor promoter activity. Exp. Cell Res. 290, 38-48.

Dimri, G.P., Itahana, K., Acosta, M., Campisi, J., 2000. Regulation of a senescence checkpoint response by the E2F1 transcription factor and p14/ARF tumor suppressor. Mol. Cell. Biol. 20, 273-285.

Dimri, G.P., Lee, X., Basile, G., Acosta, M., Scott, G., Roskelley, C., Medrano, E.E., Linskens, M., Rubelj, I., Pereira-Smith, O.M., Peacocke, M., Campisi, J., 1995. A novel biomarker identifies senescent human cells in culture and in aging skin in vivo. Proc. Natl. Acad. Sci. U.S.A. 92, 9363-9367.

Ding, G., Franki, N., Kapasi, A.A., Reddy, K., Gibbons, N., Singhal, P.C., 2001. Tubular cell senescence and expression of TGF-beta1 and p21(WAF1/CIP1) in tubulointerstitial fibrosis of aging rats. Exp. Mol. Pathol. 70, 43-53.

Dolle, M.E., Snyder, W.K., Dunson, D.B., Vijg, J., 2002. Mutational fingerprints of aging. Nucleic Acids Res. 30, 545-549.

Donjacour, A.A., Cunha, G.R., 1991. Stromal regulation of epithelial function. Cancer Treat. Res. 53, 335-364.

Faragher, R.G., 2000. Cell senescence and human aging: where's the link? Biochem. Soc. Trans. 28, 221-226.

Finch, C.E., Ruvkun, G., 2001. The genetics of aging. Annu. Rev. Genomics Hum. Genet. 2, 435-462.

Friedberg, E.C., Wagner, R., Radman, M., 2002. Specialized DNA polymerases, cellular survival, and the genesis of mutations. Science 296, $1627-1630$.

Gire, V., Wynford-Thomas, D., 1998. Reinitiation of DNA synthesis and cell division in senescent human fibroblasts by microinjection of anti-p53 antibodies. Mol. Cell. Biol. 18, 1611-1621.

Gray, J.W., Collins, C., 2000. Genome changes and gene expression in human solid tumors. Carcinogenesis 21, 443-452.

Green, D.R., Evan, G.I., 2002. A matter of life and death. Cancer Cell 1, 1930 .

Guarente, L., Kenyon, C., 2000. Genetic pathways that regulate ageing in model organisms. Nature 408, 255-262.

Hasty, P., Campisi, J., Hoeijmakers, J., van Steeg, H., Vijg, J., 2003. Aging and genome maintenance: lessons from the mouse? Science 299, 13551359.

Hayflick, L., 1965. The limited in vitro lifetime of human diploid cell strains. Exp. Cell Res. 37, 614-636.

Hickman, E.S., Moroni, M.C., Helin, K., 2002. The role of p53 and pRB in apoptosis and cancer. Curr. Opin. Genet. Dev. 12, 60-66.
Hofseth, L.J., Hussain, S.P., Harris, C.C., 2004. p53: 25 years after its discovery. Trends Pharmacol. Sci. 25, 177-181.

Holzenberger, M., Dupont, J., Ducos, B., Leneuve, P., Geloen, A., Even, P.C., Cervera, P., Le Bouc, Y., 2003. IGF-1 receptor regulates lifespan and resistance to oxidative stress in mice. Nature 421, 182-187.

Itahana, K., Dimri, G., Campisi, J., 2001. Regulation of cellular senescence by p53. Eur. J. Biochem. 268, 2784-2791

Itahana, K., Zou, Y., Itahana, Y., Martinez, J.L., Beausejour, C., Jacobs, J.J., Van Lohuizen, M., Band, V., Campisi, J., Dimri, G.P., 2003. Control of the replicative life span of human fibroblasts by p16 and the polycomb protein Bmi-1. Mol. Cell. Biol. 23, 389-401.

Jacobs, J.J., Kieboom, K., Marino, S., DePinho, R.A., van Lohuizen, M., 1999. The oncogene and Polycomb-group gene bmi-1 regulates cell proliferation and senescence through the ink4a locus. Nature 397, 164 168.

Joaquin, A.M., Gollapudi, S., 2001. Functional decline in aging and disease: a role for apoptosis. J. Am. Geriatr. Soc. 49, 1234-1240.

Kim, S.H., Kaminker, P., Campisi, J., 2002. Telomeres, aging and cancer: in search of a happy ending. Oncogene 21, 503-511.

Kinzler, K.W., Vogelstein, B., 1997. Cancer susceptibility genes: gatekeepers and caretakers. Nature 386, 761-763.

Kirkwood, T.B., Austad, S.N., 2000. Why do we age? Nature 408, 233-238.

Knudson, A.G., 2000. Chasing the cancer demon. Annu. Rev. Genet. 34, 119.

Krtolica, A., Campisi, J., 2002. Cancer and aging: a model for the cancer promoting effects of the aging stroma. Int. J. Biochem. Cell Biol. 34, 1401-1414

Krtolica, A., Parrinello, S., Lockett, S., Desprez, P., Campisi, J., 2001. Senescent fibroblasts promote epithelial cell growth and tumorigenesis: a link between cancer and aging. Proc. Natl. Acad. Sci. U.S.A. 98, 12072-12077.

Kunkel, T.A., Bebenek, K., 2000. DNA replication fidelity. Annu. Rev. Biochem. 69, 497-529.

Levy, M.Z., Allsopp, R.C., Futcher, A.B., Greider, C.W., Harley, C.B., 1992. Telomere end-replication problem and cell aging. J. Mol. Biol. 225, 951-960.

Liotta, L.A., Kohn, E.C., 2001. The microenvironment of the tumour-host interface. Nature 411, 375-379.

Lundberg, A.S., Hahn, W.C., Gupta, P., Weinberg, R.A., 2000. Genes involved in senescence and immortalization. Curr. Opin. Cell Biol. $12,705-709$

Maier, B., Gluba, W., Bernier, B., Turner, T., Mohammad, K., Guise, T., Sutherland, A., Thorner, M., Scrable, H., 2004. Modulation of mammalian life span by the short isoform of p53. Genes Dev. 18, 306-319.

Martens, J.W., Sieuwerts, A.M., Vries, J.B., Bosma, P.T., Swiggers, S.J., Klijn, J.G., Foekens, J.A., 2003. Aging of stromal-derived human breast fibroblasts might contribute to breast cancer progression. Thromb. Haemost. 89, 393-404

Martin, G.M., 1966. Somatic mutagenesis and antimutagenesis in aging research. Mutat. Res. 350, 35-41.

Martin, J.A., Buckwalter, J.A., 2003. The role of chondrocyte senescence in the pathogenesis of osteoarthritis and in limiting cartilage repair. J. Bone Joint Surg. Am. 85, 106-110.

McConnell, B.B., Starborg, M., Brookes, S., Peters, G., 1998. Inhibitors of cyclin-dependent kinases induce features of replicative senescenceo in early passage human diploid fibroblasts. Curr. Biol. 8, 351-354.

Melk, A., Kittikowit, W., Sandhu, I., Halloran, K.M., Grimm, P., Schmidt, B.M., Halloran, P.F., 2003. Cell senescence in rat kidneys in vivo increases with growth and age despite lack of telomere shortening. Kidney Int. 63, 2134-2143.

Miller, R.A., 1991. Gerontology as oncology: research on aging as a key to the understanding of cancer. Cancer 68, 2496-2501.

Mishima, K., Handa, J.T., Aotaki-Keen, A., Lutty, G.A., Morse, L.S., Hjelmeland, L.M., 1999. Senescence-associated beta-galactosidase histochemistry for the primate eye. Invest. Ophthalmol. Vis. Sci. 40, 15901593. 
Narita, M., Nunez, S., Heard, E., Narita, M., Lin, A.W., Hearn, S.A., Spector, D.L., Hannon, G.J., Lowe, S.W., 2003. Rb-mediated heterochromatin formation and silencing of E2F target genes during cellular senescence. Cell 113, 703-716.

Ogryzko, V.V., Hirai, T.H., Russanova, V.R., Barbie, D.A., Howard, B.H., 1996. Human fibroblast commitment to a senescence-like state in response to histone deacetylase inhibitors is cell cycle dependent. Mol. Cell. Biol. 16, 5210-5218.

Olumi, A.F., Grossfeld, G.D., Hayward, S.W., Carroll, P.R., Tlsty, T.D., Cunha, G.R., 1999. Carcinoma-associated fibroblasts direct tumor progression of initiated human prostatic epithelium. Cancer Res. 59, 5002-5011.

Paradis, V., Youssef, N., Dargere, D., Ba, N., Bonvoust, F., Bedossa, P., 2001. Replicative senescence in normal liver, chronic hepatitis C, and hepatocellular carcinomas. Hum. Pathol. 32, 327-332.

Park, C.C., Bissell, M.J., Barcellos-Hoff, M.H., 2000. The influence of the microenvironment on the malignant phenotype. Mol. Med. Today 6, 324-329.

Parrinello, S., Coppe, J.P., Krtolica, A., Campisi, J., Stromal-epithelial interactions in aging and cancer: senescent fibroblasts can alter epithelial cell differentiation, submitted for publication.

Pendergrass, W.R., Lane, M.A., Bodkin, N.L., Hansen, B.C., Ingram, D.K., Roth, G.S., Yi, L., Bin, H., Wolf, N.S., 1999. Cellular proliferation potential during aging and caloric restriction in rhesus monkeys (Macaca mulatta). J. Cell. Physiol. 180, 123-130.

Prives, C., Hall, P.A., 1999. The p53 pathway. J. Pathol. 187, 112-126.

Reddel, R.R., 2000. The role of senescence and immortalization in carcinogenesis. Carcinogenesis 21, 477-484.

Reed, J.C., 1999. Mechanisms of apoptosis in avoidance of cancer. Curr. Opin. Oncol. 11, 68-75.

Rincon, M., Muzumdar, R., Atzmon, G., Barzilai, N., 2004. The paradox of the insulin/IGF-1 signaling pathway in longevity. Mech. Ageing Dev. 125, 397-403.

Robles, S.J., Adami, G.R., 1998. Agents that cause DNA double strand breaks lead to p16INK4a enrichment and the premature senescence of normal fibroblasts. Oncogene 16, 1113-1123.

Rose, M.R., 1991. The Evolutionary Biology of Aging. Oxford University Press, Oxford.

Ryan, K.M., Phillips, A.C., Voudsen, K.H., 2001. Regulation and function of the p53 tumor suppressor protein. Curr. Opin. Cell Biol. 13, 332-337.

Sager, R., 1991. Senescence as a mode of tumor suppression. Environ. Health Perspect. 93, 59-62.

Serrano, M., Lin, A.W., McCurrach, M.E., Beach, D., Lowe, S.W., 1997. Oncogenic ras provokes premature cell senescence associated with accumulation of p53 and p16INK4a. Cell 88, 593-602.

Shay, J.W., Wright, W.E., 2001. Telomeres and telomerase: implications for cancer and aging. Radiat. Res. 155, 188-193.

Shay, J.W., Wright, W.R., Werbin, H., 1991. Defining the molecular mechanisms of human cell immortalization. Biochim. Biophys. Acta 1071, 1-7.

Shekhar, M.P., Werdell, J., Santner, S.J., Pauley, R.J., Tait, L., 2001. Breast stroma plays a dominant regulatory role in breast epithelial growth and differentiation: implications for tumor development and progression. Cancer Res. 61, 1320-1326.

Sherr, C.J., 1998. Tumor surveillance via the ARF-p53 pathway. Genes Dev. 12, 2891-2984.

Simpson, A.J., Camargo, A.A., 1998. Evolution and the inevitability of human cancer. Sem. Cancer Biol. 8, 439-445.

Skobe, M., Fusenig, N.E., 1998. Tumorigenic conversion of immortal human keratinocytes through stromal activation. Proc. Natl. Acad. Sci. U.S.A. 95, 1050-1055.

Smith, J.R., Pereira-Smith, O.M., 1996. Replicative senescence: implications for in vivo aging and tumor suppression. Science 273, 63-67.

Stanley, A., Osler, T., 2001. Senescence and the healing rates of venous ulcers. J. Vasc. Surg. 33, 1206-1211.

Sugrue, M.M., Shin, D.Y., Lee, S.W., Aaronson, S.A., 1997. Wild-type p53 triggers a rapid senescence program in human tumor cells lacking functional p53. Proc. Natl. Acad. Sci. U.S.A. 94, 96489653.

Thompson, L.H., Schild, D., 2002. Recombinational DNA repair and human disease. Mutat. Res. 509, 49-78.

Tlsty, T.D., Hein, P.W., 2001. Know thy neighbor: stromal cells can contribute oncogenic signals. Curr. Opin. Genet. Dev. 11, 54-59.

Tyner, S.D., Venkatachalam, S., Choi, J., Jones, S., Ghebranious, N., Ingelmann, H., Lu, X., Soron, G., Cooper, B., Brayton, C., Park, S.H., Thompson, T., Karsenty, G., Bradley, A., Donehower, L., 2002. p53 mutant mice that display early aging-associated phenotypes. Nature $415,45-53$.

van Brabant, A.J., Stan, R., Ellis, N.A., 2000. DNA helicases, genomic instability, and human genetic disease. Annu. Rev. Genomics Hum. Genet. 1, 409-459.

Vasile, E., Tomita, Y., Brown, L.F., Kocher, O., Dvorak, H.F., 2001. Differential expression of thymosin beta- 10 by early passage and senescent vascular endothelium is modulated by VPF/VEGF: evidence for senescent endothelial cells in vivo at sites of atherosclerosis. FASEB J. 15, 458-466.

Vijg, J., Dolle, M.E., 2002. Large genome rearrangements as a primary cause of aging. Mech. Ageing Dev. 123, 907-915.

Wahl, G.M., Carr, A.M., 2001. The evolution of diverse biological responses to DNA damage: insights from yeast and p53. Nature Cell Biol. 3, $277-$ 286.

Walker, D.W., McColl, G., Jenkins, N.L., Harris, J., Lithgow, G.J., 2000 Evolution of lifespan in C. elegans. Nature 405, 296-297.

Weinstein, B.S., Ciszek, D., 2002. The reserve capacity hypothesis: evolutionary origins and modern implications between tumor suppression and tissue repair. Exp. Geront. 37, 615-627.

Williams, G.C., 1957. Pleiotropy, natural selection, and the evolution of senescence. Evolution 11, 398-411.

Wright, W.E., Shay, J.W., 2001. Cellular senescence as a tumor-protection mechanism: the essential role of counting. Curr. Opin. Genet. Dev. 11, 98-103.

Zhang, Y., Herman, B., 2002. Ageing and apoptosis. Mech. Ageing Dev. $123,245-260$.

Zhu, J., Woods, D., McMahon, M., Bishop, J.M., 1998. Senescence of human fibroblasts induced by oncogenic raf. Genes Dev. 12, 2997-3007. 